\section{LESSON 7}

MARGINS:

Left, Pica 20; Elite 30;

Right, moved out of the way.

PAPER: Against the paper guide at 0 on the scale on the left. Turn up 7 single lines.

Check the 'ready-to-type' position: feet, body, fingers and eyes.

\section{Warm up}

Bounce your right thumb off the space bar.

Keep your eyes on the copy.

Return the carriage smartly.

Tap the keys sharply.

m key

Practise the reach until you can type $m$ with the $j$ finger without looking down.

Keep the ;lk fingers in position while typing $\mathrm{m}$.

Keep your eyes on the copy.

Tap the keys sharply.

c key

Practise the reach until you can type $c$ with the $d$ finger without looking down.

Keep the as fingers in position when typing $c$.

Keep your eyes on the copy.

Tap the keys sharply.

Return the carriage smartly.

\section{Consolidation}

Check the 'ready-to-type' position. Tap the keys sharply.

Bounce your right thumb off the space bar.

Keep your eyes on the copy.

Try to type each line in $1 / 2$ minute.

Use the paper release lever when taking the paper out of the machine.

Centre and lock the carriage.

Cover the machine.

UNIT 2

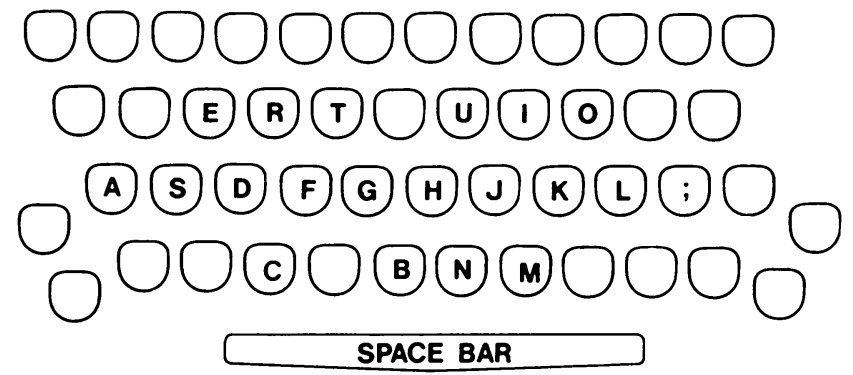

Remember to leave a blank line between sections.

Type the lines indicated by your teacher 3 times each.

1. if it is as he did it is not on to do it

2. he had his usual jaffa orange drink then

3. just then the sun began to shine on her:

4. it shrank as he said and it just fit dad

5. the oil is just too thin for the big end

Type this line as many times as your teacher asks.

6. jjj jmj jmj mjm jmm jmj mjm jmm jmjm jmj

Type the lines indicated by your teacher 3 times each.

7. a mat im him me met mu mud um rum am ham

8. man man mat mat ram ram jam jam men memo

9. melt melt milk milk them them time time;

10. some slim men mole in grim and dim mines

Type this line as many times as your teacher asks.

11. ddd ded ded ece ded dec ded cedd ded ded

Type the lines indicated by your teacher 3 times each.

12. ca cat ca can ci cit cite ic tic oc toch

13. can can cut cut act act car car cur cur:

14. cut each cat in reach a can of rich fish

15. cut his lines each time he comes across;

Type each line 3 times. Try to build up speed each time you repeat a line.

16. the skili in a team is not clear to him;

17. catch it in both hands if it falls under

18. a best mind must take care it gets fond;

19. just call and see if the mike is set on;

20. the milk cart is just along the far road

How big is a sheet of A5 typing paper?

How big is a sheet of A4 typing paper? 